\title{
Exacerbation of Dimorphous Leprosy Apparently Due to BCG Vaccination*
}

\author{
F. J. WRIGHT \\ Department of Tropical Medicine, University of Edinburgh, \\ Edinburgh, Scotland
}

\begin{abstract}
A patient whose dimorphous leprosy was clinically and bacteriologically quiescent after 4 years of treatment with dapsone, experienced a sudden reactivation of the skin lesions a month after receiving BCG vaccination, together with a slight increase in evidence of peripheral nerve damage. The reaction subsided spontaneously, dapsone treatment being continued at the same dosage as before. No other treatment was given.
\end{abstract}

The factors suspected of precipitating episodes of acute exacerbation in lepromatous and dimorphous leprosy are many and varied: any intercurrent disease, a raised temperature (from whatever cause), smallpox vaccination, a change in the hormonal state, and mental stress are among these factors. In the patient who is the subject of the following report, BCG vaccination apparently acted as the exciting cause of clinical reactivation of dimorphous leprosy lesions that had been quiescent for some time.

\section{CASE REPORT $\dagger$}

The patient was a female, 35 years of age, born (of British parents) in India, where she had remained, except for short visits to Britain, until 1965. She was married and had one child (born in 1964).

The first leprosy lesion was noticed by the patient in 1960, on the right thigh. It was diagnosed as a mycosis and treated with various fungicidal ointments. It disappeared while she was pregnant, but returned after parturition, becoming larger and more obvious. A year later, a similar lesion appeared on the right cheek. In 1965, "dimorphous leprosy" was diagnosed on clinical and histological grounds. A section of skin from the lesion on the thigh showed a "dense infiltrate of foamy cells in the superficial dermis. ... Many Myco. leprae present".

Clinical examination at this time revealed extensive annular lesions extending from the right buttock to the outer aspect of the middle-third of the right thigh. The inner border was well defined, but the outer margins faded imperceptibly into the adjacent normal skin. The colour of the actual lesions was coppery (Figs 1-3).

On the right cheek, left arm, and over the left hip small discrete hypopigmented macules, with erythematous margins, were present. Over the lower parts of both legs were numerous small and slightly raised erythematous

\footnotetext{
* Accepted for publication 18 August, 1971.

$\dagger$ This case was previously referred to in Wright, F. J. (1971) Scott. Med. J. 16, 209.
} 


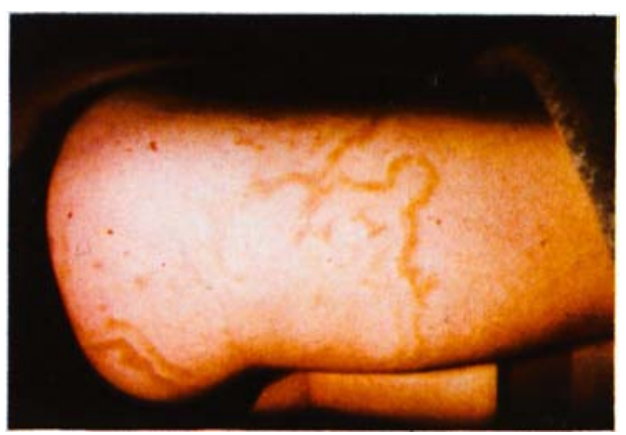

Fig. I. Original appearance of right thigh.

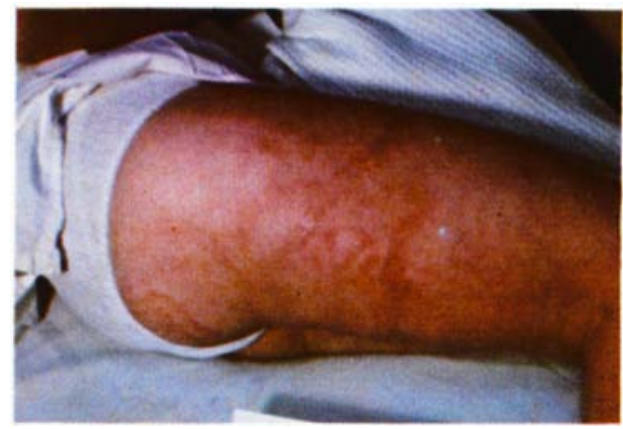

lig. 2. Right thigh after reaction to BC'

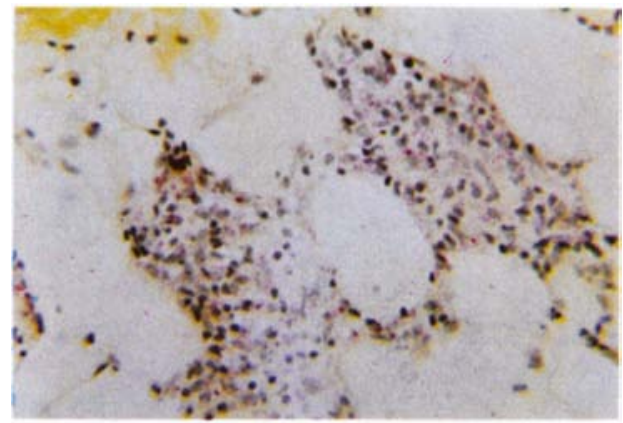

(a)

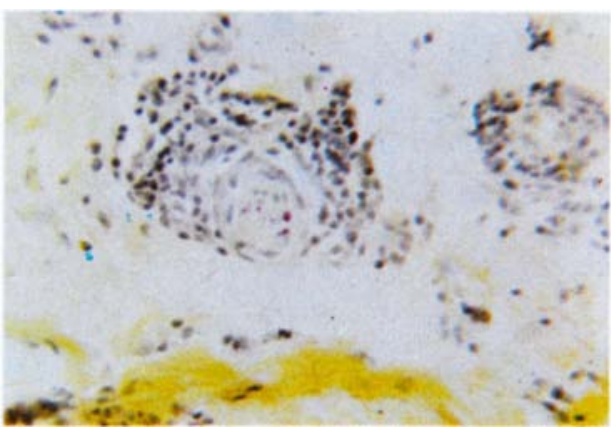

Fig. 3.(a) and (b). Histological appearance of lesions in sections from right thigh and left leg after reaction to $B C G$.

(b) 
lesions. No sensory loss was detectable in these areas. In the area enclosed by the ring-like lesion on the right thigh there was some loss of tactile sensation and of thermal appreciation and pin-prick. The peripheral nerves showed no abnormality.

\section{TREATMENT}

The lesions responded rapidly to small doses of dapsone, and the bacterioscopic findings were very satisfactory, only a few granular organisms remaining recognizable after 6 months' treatment. Treatment with dapsone was continued for a total of 4 years. By this time no trace of the skin lesions could be detected, and the only abnormalities discovered were a small area of residual skin anaesthesia on the right thigh, and enlarged dorsalis pedis nerves on both feet.

\section{FURTHER HISTORY}

The patient's husband having been diagnosed as suffering from pulmonary tuberculosis, the patient was tested twice with tuberculin (Heaf) and was found to be negative.

On 15 January, 1970, she was given BCG vaccination in the left deltoid region. On 15 February, 1970, the lesion on the right cheek re-appeared as a red ring, and the skin over the right thigh and leg (at the sites of the former lesions) became red and raised. The patient noticed tingling in the left foot. Clinical examination 4 days later confirmed these findings: the lesions seen were very similar to the appearance noted 4 years previously. There was no increase in the area or degree of sensory loss in the skin.

\section{LABORATORY FINDINGS}

Examination of the blood disclosed no abnormality. The erythrocyte sedimentation rate was $3 \mathrm{~mm}$ after $1 \mathrm{~h}$.

\section{TREATMENT}

The patient continued taking dapsone by mouth in a dose of $100 \mathrm{mg}$ twice weekly. The paraesthesiae on the outer side of the left foot persisted, but no sensory loss was at first demonstrable by standard tests.

\section{HISTOLOGICAL REPORT}

"Cellular infiltrate along some of the neurovascular pathways in the deep and middle dermis . . . cells are histiocytes, lymphocytes and a few plasma cells. A few small clusters of irregularly staining acid-fast bacilli in the arrector pili muscles ... no evidence of bacillary viability" (specimen rather shrunken).

\section{FURTHER PROGRESS}

By 9 June, 1970 the skin lesions had almost completely faded, and on 24 November, 1970 only a faint trace of them remained. Superficial sensory loss over a small area on the outer side of the left foot persisted and the skin over the dorsum of the 4 th and 5 th left toes now showed a similar pattern of sensory loss. 